\title{
Orbit Determination with the two-body Integrals. III
}

\author{
G. F. Gronchi; G. Baù \\ Dipartimento di Matematica, Università di Pisa, \\ Largo B. Pontecorvo, 5, Pisa, Italy
}

October 9, 2018

\begin{abstract}
We present the results of our investigation on the use of the twobody integrals to compute preliminary orbits by linking too short arcs of observations of celestial bodies. This work introduces a significant improvement with respect to the previous papers on the same subject [3], [4]. Here we find a univariate polynomial equation of degree 9 in the radial distance $\rho$ of the orbit at the mean epoch of one of the two arcs. This is obtained by a combination of the algebraic integrals of the two-body problem. Moreover, the elimination step, which in [3, 4] was done by resultant theory coupled with the discrete Fourier transform, is here obtained by elementary calculations. We also show some numerical tests to illustrate the performance of the new algorithm.
\end{abstract}

\section{Introduction}

This paper is related to the research started in [3, [4], where some first integrals of the two-body problem were used to write polynomial equations for the linkage problem with optical observations of asteroids and space debris.

*gronchi@dm. unipi.it

†bagiugio@gmail.com

${ }_{\ddagger}^{\ddagger}$ maro@mail.dm.unipi.it 
In [3] the authors consider polynomial equations with total degree 48, that are consequences of the conservation of angular momentum and energy, and they propose two methods to search for all the solutions using algebraic elimination theory. The same equations were first introduced in [7], but their algebraic character was not fully exploited at that time. In [4] the authors introduce for the same purpose new polynomial equations, with total degree 20, using the angular momentum integral and a projection of Laplace-Lenz vector along a suitable direction. Both in [3] and in [4] the number of the considered equations is equal to the number of unknowns.

Here we improve significantly the previous results by writing an overdetermined polynomial system (more equations than unknowns) which is proved to be generically consistent, i.e. the set of solutions in the complex field is not empty. For generic values of the data, by variable elimination, we obtain a system of two univariate polynomials of degree 10 with a greatest common divisor of degree 9 .

We also discuss the case where the oblateness of the Earth is relevant, so that we add the perturbation of the $J_{2}$ term to the Keplerian potential. In [2] and [4] this problem was faced by an iterative scheme, writing at each step polynomial equations with the same algebraic structure as in the unperturbed case (without $J_{2}$ effect). In this paper, the overdetermined system that can be written following the steps of the unperturbed case has the same algebraic structure but is generically inconsistent. However, we can use the iterative scheme mentioned above by neglecting one polynomial of the system.

This paper is organized as follows. After recalling the linkage problem and some preliminaries on the two-body integrals (Sections 2, 3), in Section 4 we discuss the polynomial equations that can be written for this problem, including the overdetermined system (9) which is the object of this work. In Section 5 we show how the elimination steps can be carried out, and we prove the consistency of system (9). In Section 6 we discuss the spurious solutions of (9), and illustrate two methods to discard them. Section 7 is devoted to the linkage problem with the $J_{2}$ effect. Some numerical tests are presented in Section 8. In the Appendix we discuss a simple way to filter the pairs of attributables to be linked with this algorithm. 


\section{Linkage of too short arcs}

We consider objects moving in a central force field. Let us fix an inertial reference frame, with the origin at the center of attraction $O$, which is the center of the Sun (Earth) in the asteroid (space debris) case. Assume the position $\mathbf{q}$ and velocity $\dot{\mathbf{q}}$ of the observer are known functions of time. We describe the position of the observed body as the sum

$$
\mathbf{r}=\mathbf{q}+\rho \mathbf{e}^{\rho}
$$

with $\rho$ the topocentric distance and $\mathbf{e}^{\rho}$ the line of sight unit vector. We choose spherical coordinates $(\alpha, \delta, \rho) \in[-\pi, \pi) \times(-\pi / 2, \pi / 2) \times \mathbb{R}^{+}$, so that

$$
\mathbf{e}^{\rho}=(\cos \delta \cos \alpha, \cos \delta \sin \alpha, \sin \delta) .
$$

A typical choice for $\alpha, \delta$ is right ascension and declination. The velocity vector is

$$
\dot{\mathbf{r}}=\dot{\mathbf{q}}+\dot{\rho} \mathbf{e}^{\rho}+\rho\left(\dot{\alpha} \cos \delta \mathbf{e}^{\alpha}+\dot{\delta} \mathbf{e}^{\delta}\right), \quad \dot{\rho}, \dot{\alpha}, \dot{\delta} \in \mathbb{R}, \rho \in \mathbb{R}^{+},
$$

where $\dot{\rho}, \rho \dot{\alpha} \cos \delta, \rho \dot{\delta}$ are the components of the velocity, relative to the observer, in the (positively oriented) orthonormal basis $\left\{\mathbf{e}^{\rho}, \mathbf{e}^{\alpha}, \mathbf{e}^{\delta}\right\}$, with

$$
\mathbf{e}^{\alpha}=(\cos \delta)^{-1} \frac{\partial \mathbf{e}^{\rho}}{\partial \alpha}, \quad \mathbf{e}^{\delta}=\frac{\partial \mathbf{e}^{\rho}}{\partial \delta} .
$$

Let $\left(t_{i}, \alpha_{i}, \delta_{i}\right)$ with $i=1 \ldots m, m \geq 2$, be a short arc of optical observations of a moving body, made from the same station. If $m \geq 3$, we can compute $\alpha, \delta, \dot{\alpha}, \dot{\delta}, \ddot{\alpha}, \ddot{\delta}$ at the mean time $\bar{t}=\frac{1}{m} \sum_{i=1}^{m} t_{i}$ by a quadratic fit. From these quantities we can try to compute a preliminary orbit. When the second derivatives are not reliable due to errors in the observations (or not available, if $m=2$ ) we speak of a too short arc (TSA) and, to compute a preliminary orbit, we have to add information coming from other arcs of observations. This is a typical identification problem, see [6].

In any case, it is possible to compute an attributable

$$
\mathcal{A}=(\alpha, \delta, \dot{\alpha}, \dot{\delta}) \in[-\pi, \pi) \times(-\pi / 2, \pi / 2) \times \mathbb{R}^{2},
$$

representing the angular position and velocity of the body at epoch $\bar{t}$ (see [5], [3]). The radial distance and velocity $\rho, \dot{\rho}$ are completely undetermined and are the missing quantities to define an orbit for the body.

In this paper we deal with the linkage problem, that is to join together two TSAs of observations to form an orbit fitting all the data. 


\section{First integrals of Kepler's motion}

We consider the first integrals of the equation of Kepler's problem

$$
\ddot{\mathbf{r}}=-\frac{\mu}{|\mathbf{r}|^{3}} \mathbf{r}
$$

as functions of the unknowns $\rho, \dot{\rho}$. The angular momentum is the polynomial vector

$$
\mathbf{c}(\rho, \dot{\rho})=\mathbf{r} \times \dot{\mathbf{r}}=\mathbf{D} \dot{\rho}+\mathbf{E} \rho^{2}+\mathbf{F} \rho+\mathbf{G},
$$

with

$$
\mathbf{D}=\mathbf{q} \times \mathbf{e}^{\rho}, \quad \mathbf{E}=\mathbf{e}^{\rho} \times \mathbf{e}^{\perp}, \quad \mathbf{F}=\mathbf{q} \times \mathbf{e}^{\perp}+\mathbf{e}^{\rho} \times \dot{\mathbf{q}}, \quad \mathbf{G}=\mathbf{q} \times \dot{\mathbf{q}},
$$

where we have set

$$
\mathbf{e}^{\perp}=\dot{\alpha} \cos \delta \mathbf{e}^{\alpha}+\dot{\delta} \mathbf{e}^{\delta}
$$

The expression of the energy is

$$
\mathcal{E}(\rho, \dot{\rho})=\frac{1}{2}|\dot{\mathbf{r}}|^{2}-\frac{\mu}{|\mathbf{r}|},
$$

where

$$
\begin{aligned}
|\mathbf{r}| & =\left(\rho^{2}+|\mathbf{q}|^{2}+2 \rho \mathbf{q} \cdot \mathbf{e}^{\rho}\right)^{1 / 2}, \\
|\dot{\mathbf{r}}|^{2} & =\dot{\rho}^{2}+\left|\mathbf{e}^{\perp}\right|^{2} \rho^{2}+2 \dot{\mathbf{q}} \cdot \mathbf{e}^{\rho} \dot{\rho}+2 \dot{\mathbf{q}} \cdot \mathbf{e}^{\perp} \rho+|\dot{\mathbf{q}}|^{2} .
\end{aligned}
$$

The Laplace-Lenz vector $\mathbf{L}$ is given by

$$
\mu \mathbf{L}(\rho, \dot{\rho})=\dot{\mathbf{r}} \times \mathbf{c}-\mu \frac{\mathbf{r}}{|\mathbf{r}|}=\left(|\dot{\mathbf{r}}|^{2}-\frac{\mu}{|\mathbf{r}|}\right) \mathbf{r}-(\dot{\mathbf{r}} \cdot \mathbf{r}) \dot{\mathbf{r}}
$$

with $|\mathbf{r}|,|\dot{\mathbf{r}}|^{2}$ as in (2), (3), and

$$
\dot{\mathbf{r}} \cdot \mathbf{r}=\rho \dot{\rho}+\mathbf{q} \cdot \mathbf{e}^{\rho} \dot{\rho}+\left(\dot{\mathbf{q}} \cdot \mathbf{e}^{\rho}+\mathbf{q} \cdot \mathbf{e}^{\perp}\right) \rho+\dot{\mathbf{q}} \cdot \mathbf{q} .
$$

Moreover, the following relations hold for all $\rho, \dot{\rho}$ :

$$
\mathbf{c} \cdot \mathbf{L}=0, \quad \mu^{2}|\mathbf{L}|^{2}=\mu^{2}+2 \mathcal{E}|\mathbf{c}|^{2} .
$$

Expressions (1), (4) are algebraic, but not polynomial, in $\rho, \dot{\rho}$. However, we can introduce a new variable $u \in \mathbb{R}$, together with the relation $|\mathbf{r}|^{2} u^{2}=\mu^{2}$ and we obtain

$$
\mathcal{E}=\frac{1}{2}|\dot{\mathbf{r}}|^{2}-u, \quad \mu \mathbf{L}=\left(|\dot{\mathbf{r}}|^{2}-u\right) \mathbf{r}-(\dot{\mathbf{r}} \cdot \mathbf{r}) \dot{\mathbf{r}}
$$


that are polynomials in $\rho, \dot{\rho}, u$.

For later reference we also introduce the quantity

$$
\mathbf{K}=\frac{1}{2}|\dot{\mathbf{r}}|^{2} \mathbf{r}-(\dot{\mathbf{r}} \cdot \mathbf{r}) \dot{\mathbf{r}}
$$

\section{Polynomial equations for the linkage}

We use the notation above, with index 1 or 2 referring to the epoch. Let

$$
\mathcal{A}_{j}=\left(\alpha_{j}, \delta_{j}, \dot{\alpha}_{j}, \dot{\delta}_{j}\right), \quad j=1,2
$$

be two attributables at epochs $\bar{t}_{j}$. We consider the polynomial system

$$
\mathbf{c}_{1}=\mathbf{c}_{2}, \quad \mathbf{L}_{1}=\mathbf{L}_{2}, \quad \mathcal{E}_{1}=\mathcal{E}_{2}, \quad u_{1}^{2}\left|\mathbf{r}_{1}\right|^{2}=\mu^{2}, \quad u_{2}^{2}\left|\mathbf{r}_{2}\right|^{2}=\mu^{2},
$$

in the 6 unknowns

$$
\left(\rho_{1}, \rho_{2}, \dot{\rho}_{1}, \dot{\rho}_{2}, u_{1}, u_{2}\right) .
$$

System (7) is defined by the vector of parameters

$$
\left(\mathcal{A}_{1}, \mathcal{A}_{2}, \mathbf{q}_{1}, \mathbf{q}_{2}, \dot{\mathbf{q}}_{1}, \dot{\mathbf{q}}_{2}\right),
$$

and we shall discuss properties which hold for generic values of them. Moreover, (7) is composed by 9 equations with 6 unknowns. However, due to relations (5), 2 equations can be considered as consequences of the others, so that we are left with a system of 7 equations with 6 unknowns.

Assume the attributables $\mathcal{A}_{1}, \mathcal{A}_{2}$ refer to the same observed body, the two-body dynamics is perfectly respected, and there are no observing errors. Then the set of solutions of (7) is not empty. Taking into account the observational errors, and the fact that the two-body motion is only an approximation, system (7) turns out to be generically inconsistent, i.e. it has no solution in $\mathbb{C}$.

We search for a polynomial system, consequence of (7), which is generically consistent, with a finite number of solutions in $\mathbb{C}$, and which leads by elimination to a univariate polynomial equation of the lowest degree possible.

Introducing relations $u_{j}^{2}\left|\mathbf{r}_{j}\right|^{2}=\mu^{2}(j=1,2)$ for the auxiliary variables $u_{1}$, $u_{2}$ corresponds to the squaring operations, used in [3], [4] to bring the selected algebraic system in the variables $\left(\rho_{1}, \rho_{2}, \dot{\rho}_{1}, \dot{\rho}_{2}\right)$ into a polynomial form. Since these operations are responsible of the high total degree of the resulting 
polynomial systems (48 and 20 respectively), in writing the new equations we try to cancel the dependence on both $u_{1}, u_{2}$ by algebraic manipulations of the conservation laws. First we shall consider the intermediate system

$$
\mathbf{c}_{1}=\mathbf{c}_{2}, \quad \mu\left(\mathbf{L}_{1}-\mathbf{L}_{2}\right)=\left(\mathcal{E}_{1}-\mathcal{E}_{2}\right) \mathbf{r}_{2}, \quad u_{1}^{2}\left|\mathbf{r}_{1}\right|^{2}=\mu^{2},
$$

where $u_{2}$ does not appear, which is still inconsistent; then we shall take into account the system

$$
\mathbf{c}_{1}=\mathbf{c}_{2}, \quad\left(\mathbf{K}_{1}-\mathbf{K}_{2}\right) \times\left(\mathbf{r}_{1}-\mathbf{r}_{2}\right)=\mathbf{0},
$$

where also $u_{1}$ does not appear, whose consistency is proven in the next section.

\section{Elimination of variables}

In this section we show that generically system $(9)$ is consistent. In particular, by elimination of variables, we shall end up with two univariate polynomials of degree 10 in the range $\rho_{2}$, whose greatest common divisor generically has degree 9. A similar procedure can be carried out by eliminating all the variables but $\rho_{1}$.

\subsection{Angular momentum equations}

The conservation of angular momentum gives us 3 polynomial equations that are linear in $\dot{\rho}_{1}, \dot{\rho}_{2}$, and quadratic in $\rho_{1}, \rho_{2}$. Therefore, it is natural to use these equations to eliminate the radial velocities, as done in [3], [4]. These equations can be written as

$$
\mathbf{D}_{1} \dot{\rho}_{1}-\mathbf{D}_{2} \dot{\rho}_{2}=\mathbf{J}\left(\rho_{1}, \rho_{2}\right),
$$

with $\mathbf{J}$ a vector whose components are quadratic polynomials in $\rho_{1}, \rho_{2}$. Following [3] we project (10) onto the vectors $\mathbf{D}_{2} \times\left(\mathbf{D}_{1} \times \mathbf{D}_{2}\right)$ and $\mathbf{D}_{1} \times\left(\mathbf{D}_{1} \times \mathbf{D}_{2}\right)$ and obtain $\dot{\rho}_{1}, \dot{\rho}_{2}$ as quadratic polynomials in $\rho_{1}, \rho_{2}$. With these expressions of $\dot{\rho}_{1}, \dot{\rho}_{2}$ we have

$$
\boldsymbol{\Delta}_{c} \times\left(\mathbf{D}_{1} \times \mathbf{D}_{2}\right)=\mathbf{0},
$$

with $\boldsymbol{\Delta}_{c}=\mathbf{c}_{1}-\mathbf{c}_{2}$, whatever the values of $\rho_{1}, \rho_{2}$. The projection of 110 onto $\mathbf{D}_{1} \times \mathbf{D}_{2}$ allows us to eliminate the variables $\dot{\rho}_{1}, \dot{\rho}_{2}$ and yields

$$
q\left(\rho_{1}, \rho_{2}\right)=q_{2,0} \rho_{1}^{2}+q_{1,0} \rho_{1}+q_{0,2} \rho_{2}^{2}+q_{0,1} \rho_{2}+q_{0,0},
$$


where the coefficients $q_{i, j}$ depend only on the attributables and on the position and velocity of the observer at epochs $\bar{t}_{1}, \bar{t}_{2}$.

In the following we shall consider the quantities introduced in Section 3 as function of $\rho_{1}, \rho_{2}$ only, by the elimination of $\dot{\rho}_{1}, \dot{\rho}_{2}$ just recalled.

\subsection{Bivariate equations for the linkage}

By subtracting $\mathcal{E}_{2} \mathbf{r}_{2}$ to both members of the Laplace-Lenz equation, and using the conservation of energy, we obtain

$$
\mu \mathbf{L}_{1}-\mathcal{E}_{1} \mathbf{r}_{2}=\mu \mathbf{L}_{2}-\mathcal{E}_{2} \mathbf{r}_{2}
$$

Equation (13) can be written

$$
\boldsymbol{\Delta}_{K}+\left(\frac{1}{2}\left|\dot{\mathbf{r}}_{1}\right|^{2}-u_{1}\right) \boldsymbol{\Delta}_{r}=\mathbf{0}
$$

where we have set

$$
\boldsymbol{\Delta}_{K}=\mathbf{K}_{1}-\mathbf{K}_{2}, \quad \boldsymbol{\Delta}_{r}=\mathbf{r}_{1}-\mathbf{r}_{2},
$$

with $\mathbf{K}$ as in (6). Note that the variable $u_{2}$ does not appear in (14).

We can also eliminate $u_{1}$ by cross product with $\boldsymbol{\Delta}_{r}$ :

$$
\Delta_{K} \times \Delta_{r}=\mathbf{0}
$$

For brevity we set $\boldsymbol{\xi}=\boldsymbol{\Delta}_{K} \times \boldsymbol{\Delta}_{r}$, and we note that

$$
\boldsymbol{\xi}=\frac{1}{2}\left(\left|\dot{\mathbf{r}}_{2}\right|^{2}-\left|\dot{\mathbf{r}}_{1}\right|^{2}\right) \mathbf{r}_{1} \times \mathbf{r}_{2}-\left(\dot{\mathbf{r}}_{1} \cdot \mathbf{r}_{1}\right) \dot{\mathbf{r}}_{1} \times \boldsymbol{\Delta}_{r}+\left(\dot{\mathbf{r}}_{2} \cdot \mathbf{r}_{2}\right) \dot{\mathbf{r}}_{2} \times \boldsymbol{\Delta}_{r}
$$

Remark 1. By developing the expressions of $\mathbf{r}_{1} \times \mathbf{r}_{2}, \dot{\mathbf{r}}_{1} \times \boldsymbol{\Delta}_{r}, \dot{\mathbf{r}}_{2} \times \boldsymbol{\Delta}_{r}$ as polynomials in $\rho_{1}, \rho_{2}$ we obtain that the monomials in $\boldsymbol{\xi}$ with the highest total degree, which is 6 , are all multiplied by $\mathbf{e}_{1}^{\rho} \times \mathbf{e}_{2}^{\rho}$.

In the following section we shall prove that for generic values of the data the system

$$
q=0, \quad \xi=0
$$

is consistent, that is the set of its roots in $\mathbb{C}$ is not empty.

Note that, if $q=0$, the vector $\boldsymbol{\xi}$ is parallel to the common value $\mathbf{c}_{1}=\mathbf{c}_{2}$ of the angular momentum. 


\subsection{Consistency of the equations}

The proof of the consistency relies on some geometrical considerations. In particular it is relevant to check whether the angular momentum vector is orthogonal to the line of sight. We introduce the quantities

$$
c_{i j}=\mathbf{c}_{i} \cdot \mathbf{e}_{j}^{\rho}, \quad i, j=1,2 .
$$

More explicitly we have

$$
\begin{aligned}
c_{11} & =\mathbf{q}_{1} \times \mathbf{e}_{1}^{\rho} \cdot \mathbf{e}_{1}^{\perp} \rho_{1}+\mathbf{q}_{1} \times \mathbf{e}_{1}^{\rho} \cdot \dot{\mathbf{q}}_{1}, \\
c_{12} & =\mathbf{e}_{1}^{\rho} \times \mathbf{e}_{1}^{\perp} \cdot \mathbf{e}_{2}^{\rho} \rho_{1}^{2}+\mathbf{q}_{1} \times \mathbf{e}_{1}^{\rho} \cdot \mathbf{e}_{2}^{\rho} \dot{\rho}_{1}\left(\rho_{1}, \rho_{2}\right)+ \\
& +\left(\mathbf{e}_{1}^{\rho} \times \dot{\mathbf{q}}_{1}+\mathbf{q}_{1} \times \mathbf{e}_{1}^{\perp}\right) \cdot \mathbf{e}_{2}^{\rho} \rho_{1}+\mathbf{q}_{1} \times \dot{\mathbf{q}}_{1} \cdot \mathbf{e}_{2}^{\rho},
\end{aligned}
$$

and similar expressions for $c_{22}, c_{21}$. In particular, equations $c_{11}=0$ and $c_{22}=0$ represent straight lines in the plane $\rho_{1} \rho_{2}$, while $c_{12}=0$ and $c_{21}=0$ give conic sections, see Figure 1 .

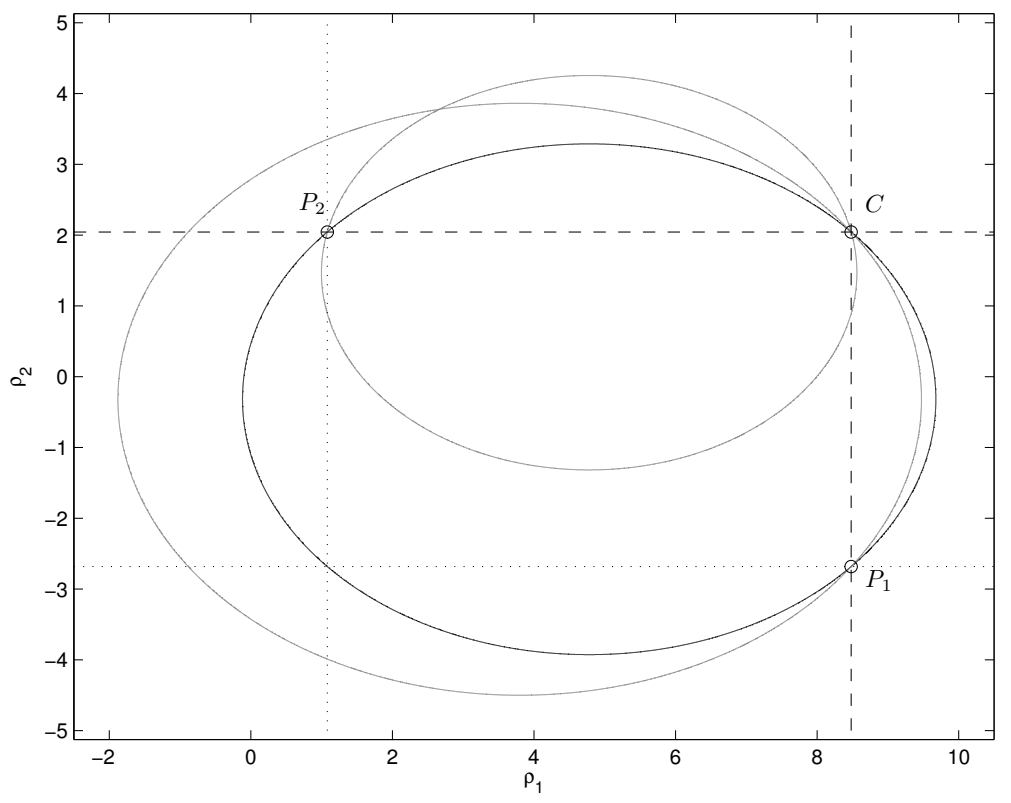

Figure 1: In the plane $\rho_{1} \rho_{2}$, for a test case, we draw the curves $q=0$ (black), $c_{12}=0$ and $c_{21}=0$ (light gray), and the straight lines $c_{11}=0, c_{22}=0$ (dashed), and $\rho_{1}=\rho_{1}^{\prime}, \rho_{2}=\rho_{2}^{\prime}$ (dotted). 
Consider the point $C=\left(\rho_{1}^{\prime \prime}, \rho_{2}^{\prime \prime}\right)$ defined by $c_{11}=c_{22}=0$, so that

$$
\rho_{1}^{\prime \prime}=\frac{\mathbf{q}_{1} \times \dot{\mathbf{q}}_{1} \cdot \mathbf{e}_{1}^{\rho}}{\mathbf{e}_{1}^{\rho} \times \mathbf{e}_{1}^{\perp} \cdot \mathbf{q}_{1}}, \quad \rho_{2}^{\prime \prime}=\frac{\mathbf{q}_{2} \times \dot{\mathbf{q}}_{2} \cdot \mathbf{e}_{2}^{\rho}}{\mathbf{e}_{2}^{\rho} \times \mathbf{e}_{2}^{\perp} \cdot \mathbf{q}_{2}} .
$$

In Lemma 1 we shall prove that $C$ lies on the conic $q=0$ and is the only point where both angular momenta $\mathbf{c}_{1}, \mathbf{c}_{2}$ vanish. The straight line $\rho_{2}=\rho_{2}^{\prime \prime}$ generically meets $q=0$ in another point $P_{2}=\left(\rho_{1}^{\prime}, \rho_{2}^{\prime \prime}\right)$, where the angular momenta do not vanish. Similarly, the straight line $\rho_{1}=\rho_{1}^{\prime \prime}$ generically meets $q=0$ in another point $P_{1}=\left(\rho_{1}^{\prime \prime}, \rho_{2}^{\prime}\right)$, where the angular momenta are not zero, see Figure 1. For $q=0$, the vector $\mathbf{r}_{1} \times \mathbf{r}_{2}$ gives the direction of $\mathbf{c}_{1}=\mathbf{c}_{2}$, therefore from the equations $\mathbf{r}_{1} \times \mathbf{r}_{2} \cdot \mathbf{e}_{j}^{\rho}=0, j=1,2$ we obtain

$$
\rho_{1}^{\prime}=\frac{\mathbf{q}_{1} \times \mathbf{q}_{2} \cdot \mathbf{e}_{2}^{\rho}}{\mathbf{e}_{1}^{\rho} \times \mathbf{e}_{2}^{\rho} \cdot \mathbf{q}_{2}}, \quad \rho_{2}^{\prime}=\frac{\mathbf{q}_{1} \times \mathbf{q}_{2} \cdot \mathbf{e}_{1}^{\rho}}{\mathbf{e}_{1}^{\rho} \times \mathbf{e}_{2}^{\rho} \cdot \mathbf{q}_{1}} .
$$

Note that

$$
q_{2,0}=-\mathbf{E}_{1} \cdot \mathbf{D}_{1} \times \mathbf{D}_{2}=-\left(\mathbf{e}_{1}^{\rho} \times \mathbf{e}_{1}^{\perp} \cdot \mathbf{q}_{1}\right)\left(\mathbf{e}_{1}^{\rho} \times \mathbf{e}_{2}^{\rho} \cdot \mathbf{q}_{2}\right),
$$

so that $q_{2,0} \neq 0$ implies that both $\rho_{1}^{\prime}$ and $\rho_{1}^{\prime \prime}$ are well defined.

In a similar way we obtain

$$
q_{0,2}=\mathbf{E}_{2} \cdot \mathbf{D}_{1} \times \mathbf{D}_{2}=\left(\mathbf{e}_{2}^{\rho} \times \mathbf{e}_{2}^{\perp} \cdot \mathbf{q}_{2}\right)\left(\mathbf{e}_{1}^{\rho} \times \mathbf{e}_{2}^{\rho} \cdot \mathbf{q}_{1}\right),
$$

so that $q_{0,2} \neq 0$ implies that both $\rho_{2}^{\prime}$ and $\rho_{2}^{\prime \prime}$ are well defined.

Generically we have

$$
q_{2,0}, q_{0,2} \neq 0 \text {. }
$$

Lemma 1. If (20) holds, then the point $C=\left(\rho_{1}^{\prime \prime}, \rho_{2}^{\prime \prime}\right)$ given by $c_{11}=c_{22}=0$ satisfies $q\left(\rho_{1}^{\prime \prime}, \rho_{2}^{\prime \prime}\right)=0$. Moreover, in $C$ we have $\mathbf{c}_{1}=\mathbf{c}_{2}=\mathbf{0}$ and $C$ is the unique point in the plane $\rho_{1} \rho_{2}$ where both angular momenta vanish.

Proof. Using relation $\mathbf{r}_{j} \cdot \mathbf{D}_{j}=0$ we obtain

$$
\mathbf{c}_{j} \times \mathbf{D}_{j}=-\left(\dot{\mathbf{r}}_{j} \cdot \mathbf{D}_{j}\right) \mathbf{r}_{j}, \quad j=1,2 .
$$

Moreover, condition (20) yields $\mathbf{e}_{j}^{\rho} \times \mathbf{q}_{j} \neq \mathbf{0}$, so that $\mathbf{r}_{j} \neq \mathbf{0}$. From relations

$$
\dot{\mathbf{r}}_{j} \cdot \mathbf{D}_{j}=-\mathbf{c}_{j} \cdot \mathbf{e}_{j}^{\rho}
$$


we have

$$
\mathbf{c}_{j} \times \mathbf{D}_{j}=\mathbf{0} \text { if and only if } c_{j j}=0
$$

for $j=1,2$. Therefore $c_{11}=c_{22}=0$ implies

$$
\boldsymbol{\Delta}_{c} \cdot \mathbf{D}_{1} \times \mathbf{D}_{2}=0,
$$

that together with (11) gives

$$
\mathbf{c}_{1}=\mathbf{c}_{2} .
$$

Finally, relations (21), 222) imply $\mathbf{c}_{j}=\mathbf{0}, j=1,2$. The uniqueness immediately follows from the definition of $C$.

Lemma 2. In the point $C=\left(\rho_{1}^{\prime \prime}, \rho_{2}^{\prime \prime}\right)$ generically we have $\boldsymbol{\xi} \neq \mathbf{0}$.

Proof. By Lemma 1, if (20) holds, we have $\mathbf{c}_{1}=\mathbf{c}_{2}=\mathbf{0}$ in $C$, so that

$$
\begin{aligned}
& \mu\left(\mathbf{L}_{1}-\mathbf{L}_{2}\right)-\left(\mathcal{E}_{1}-\mathcal{E}_{2}\right) \mathbf{r}_{2}=\mu\left(\frac{\mathbf{r}_{2}}{\left|\mathbf{r}_{2}\right|}-\frac{\mathbf{r}_{1}}{\left|\mathbf{r}_{1}\right|}\right)-\left(\mathcal{E}_{1}-\mathcal{E}_{2}\right) \mathbf{r}_{2} \\
= & -\mu \frac{\boldsymbol{\Delta}_{r}}{\left|\mathbf{r}_{1}\right|}+\frac{1}{2}\left(\left|\dot{\mathbf{r}}_{2}\right|^{2}-\left|\dot{\mathbf{r}}_{1}\right|^{2}\right) \mathbf{r}_{2} .
\end{aligned}
$$

Therefore we have

$$
\boldsymbol{\Delta}_{K} \times \boldsymbol{\Delta}_{r}=\frac{1}{2}\left(\left|\dot{\mathbf{r}}_{1}\right|^{2}-\left|\dot{\mathbf{r}}_{2}\right|^{2}\right) \mathbf{r}_{1} \times \mathbf{r}_{2} .
$$

We show that the right-hand side of (23) does not vanish in $C$. In fact, by projecting $\mathbf{r}_{1} \times \mathbf{r}_{2}$ onto $\mathbf{q}_{1}, \mathbf{q}_{2}$ we obtain

$$
\begin{aligned}
& \mathbf{q}_{1} \cdot \mathbf{r}_{1} \times \mathbf{r}_{2}=\rho_{1}^{\prime \prime}\left[\rho_{2}^{\prime \prime}\left(\mathbf{e}_{1}^{\rho} \times \mathbf{e}_{2}^{\rho} \cdot \mathbf{q}_{1}\right)-\mathbf{q}_{1} \times \mathbf{q}_{2} \cdot \mathbf{e}_{1}^{\rho}\right], \\
& \mathbf{q}_{2} \cdot \mathbf{r}_{1} \times \mathbf{r}_{2}=\rho_{2}^{\prime \prime}\left[\rho_{1}^{\prime \prime}\left(\mathbf{e}_{1}^{\rho} \times \mathbf{e}_{2}^{\rho} \cdot \mathbf{q}_{2}\right)-\mathbf{q}_{1} \times \mathbf{q}_{2} \cdot \mathbf{e}_{2}^{\rho}\right],
\end{aligned}
$$

and the expressions in the brackets vanish only if

$$
\rho_{1}^{\prime}=\rho_{1}^{\prime \prime}, \quad \rho_{2}^{\prime}=\rho_{2}^{\prime \prime} .
$$

Moreover, $\rho_{1}^{\prime \prime}=\rho_{2}^{\prime \prime}=0$ occurs only if $\mathbf{q}_{j} \times \dot{\mathbf{q}}_{j} \cdot \mathbf{e}_{j}^{\rho}=0, j=1,2$. Thus $\mathbf{r}_{1} \times \mathbf{r}_{2}$ generically does not vanish. Using $\mathbf{c}_{1}=0$ projected e.g. onto $\mathbf{e}_{1}^{\perp}$, we can prove that in $C$ the quantity $\left|\dot{\mathbf{r}}_{1}\right|^{2}$ depends only on the data $\mathcal{A}_{1}, \mathbf{q}_{1}, \dot{\mathbf{q}}_{1}$ 
at epoch $\bar{t}_{1}$. In fact through this relation we can find an expression for $\dot{\rho}_{1}$ depending only on $\mathcal{A}_{1}, \mathbf{q}_{1}, \dot{\mathbf{q}}_{1}$. A similar result holds for $\left|\dot{\mathbf{r}}_{2}\right|^{2}$. We observe that the $\left|\dot{\mathbf{r}}_{j}\right|^{2}$ do not vanish individually. Indeed, if relations $\mathbf{e}_{j}^{\rho} \times \mathbf{e}_{j}^{\perp} \cdot \dot{\mathbf{q}}_{j} \neq 0$, $j=1,2$ hold, then $\dot{\mathbf{r}}_{j}=\dot{\rho}_{j} \mathbf{e}_{j}^{\rho}+\rho_{j} \mathbf{e}_{j}^{\perp}+\dot{\mathbf{q}}_{j} \neq \mathbf{0}$ for any choice of $\rho_{j}, \dot{\rho}_{j}$. Therefore generically also $\left|\dot{\mathbf{r}}_{1}\right|^{2}-\left|\dot{\mathbf{r}}_{2}\right|^{2}$ does not vanish. We conclude that the point $C$ is not a solution of (17).

Lemma 3. Assume $q=0$. Then $\boldsymbol{\xi}=\mathbf{0}$ is generically equivalent to

$$
\left\{\begin{array} { l } 
{ \boldsymbol { \xi } \cdot \mathbf { e } _ { 1 } ^ { \rho } = 0 } \\
{ \mathbf { c } \cdot \mathbf { e } _ { 1 } ^ { \rho } \neq 0 }
\end{array} \quad \text { or } \quad \left\{\begin{array}{l}
\boldsymbol{\xi} \cdot \mathbf{e}_{2}^{\rho}=0 \\
\mathbf{c} \cdot \mathbf{e}_{2}^{\rho} \neq 0
\end{array} .\right.\right.
$$

Proof. Assume (24) does not hold. Clearly relations $\boldsymbol{\xi} \cdot \mathbf{e}_{1}^{\rho}=\boldsymbol{\xi} \cdot \mathbf{e}_{2}^{\rho}=0$ are necessary to have $\boldsymbol{\xi}=\mathbf{0}$. Then we have $\mathbf{c} \cdot \mathbf{e}_{1}^{\rho}=\mathbf{c} \cdot \mathbf{e}_{2}^{\rho}=0$. If 20 holds, Lemma 1 implies $\mathbf{c}_{1}=\mathbf{c}_{2}=\mathbf{0}$ and, by Lemma 2, generically we have $\boldsymbol{\xi} \neq \mathbf{0}$. Viceversa, assuming $\boldsymbol{\xi} \neq \mathbf{0}$ we obtain that each system in (24) is incompatible, because for $q=0$ we have $\boldsymbol{\xi} \times \mathbf{c}=\mathbf{0}$.

Lemma 4. Assume relation

$$
\boldsymbol{\Delta}_{q} \cdot \mathbf{e}_{1}^{\rho} \times \mathbf{e}_{2}^{\rho} \neq 0
$$

holds. Then $\boldsymbol{\xi}=\mathbf{0}$ is equivalent to

$$
\boldsymbol{\xi} \cdot \mathbf{e}_{1}^{\rho}=0 \quad \text { and } \quad \boldsymbol{\xi} \cdot \mathbf{e}_{2}^{\rho}=0 .
$$

Proof. Relation $\boldsymbol{\xi} \cdot \boldsymbol{\Delta}_{r}=0$ holds trivially. Moreover, since 25) is satisfied, the vectors $\mathbf{e}_{1}^{\rho}, \mathbf{e}_{2}^{\rho}, \boldsymbol{\Delta}_{r}$ are linearly independent.

We introduce the polynomials

$$
p_{1}=\boldsymbol{\xi} \cdot \mathbf{e}_{1}^{\rho}, \quad p_{2}=\boldsymbol{\xi} \cdot \mathbf{e}_{2}^{\rho} .
$$


By Remark 1 both $p_{1}$ and $p_{2}$ have total degree 5 in the variables $\rho_{1}, \rho_{2}$. We consider the system

$$
q=p_{1}=p_{2}=0
$$

We are now ready to state the main result.

Theorem 1. Generically, system (17) is consistent and can be reduced to a system of two univariate polynomials $\mathfrak{u}_{1}, \mathfrak{u}_{2}$ whose greatest common divisor has degree 9.

Proof. By Lemma 4 we only need to prove consistency of system (26). First we show that system (17) has at least 9 solutions. In fact Lemma 3 implies that both systems $q=p_{1}=0$ and $q=p_{2}=0$ define generically 10 points. Moreover, for $q=0$ relation $c_{11} \neq 0$ discards the points $P_{1}, C$, while relation $c_{22} \neq 0$ discards the points $P_{2}, C$. By Lemma 2 we know that generically $C$ is not a solution of $\boldsymbol{\xi}=\mathbf{0}$, hence it does not solve either $p_{1}=0$ or $p_{2}=0$. On the other hand, we have $p_{1}\left(P_{1}\right)=p_{2}\left(P_{2}\right)=0$. We show that $p_{1}\left(P_{1}\right)=0$. If $\boldsymbol{\xi}\left(P_{1}\right)=\mathbf{0}$ the results trivially holds. If $\boldsymbol{\xi}\left(P_{1}\right) \neq \mathbf{0}$, by Lemma 1 we have $\mathbf{c}\left(P_{1}\right) \neq \mathbf{0}$, so that $\mathbf{c}\left(P_{1}\right) \| \boldsymbol{\xi}\left(P_{1}\right)$, and from $c_{11}\left(P_{1}\right)=0$ we obtain $p_{1}\left(P_{1}\right)=0$. In a similar way we can prove that $p_{2}\left(P_{2}\right)=0$. Therefore we are left with 9 solutions for each system. We show that they are the same ones. In fact, by Lemma 4. solutions of (17) must satisfy $p_{1}=p_{2}=0$. Moreover, generically we have $p_{1}\left(P_{2}\right) \neq 0$ and $p_{2}\left(P_{1}\right) \neq 0$, so that both $P_{1}$ and $P_{2}$ are not solutions. Therefore we have exactly 9 solutions.

Let us consider the univariate polynomials

$$
\mathfrak{u}_{j}=\operatorname{res}\left(p_{j}, q, \rho_{1}\right), \quad j=1,2,
$$

that are the resultant of the pairs $p_{j}, q$ with respect to $\rho_{1}$ (see [1]).

The root $\rho_{2}=\rho_{2}^{\prime}$ of $\mathfrak{u}_{1}$ and the root $\rho_{2}=\rho_{2}^{\prime \prime}$ of $\mathfrak{u}_{2}$ must be discarded because they correspond to the points $P_{1}, P_{2}$ for the polynomials $p_{1}, p_{2}$ respectively.

We consider

$$
\tilde{\mathfrak{u}}_{1}=\frac{\mathfrak{u}_{1}}{\rho_{2}-\rho_{2}^{\prime}}, \quad \tilde{\mathfrak{u}}_{2}=\frac{\mathfrak{u}_{2}}{\rho_{2}-\rho_{2}^{\prime \prime}} .
$$

By the discussion above, these polynomials have degree 9 and must have the same roots: in particular, up to a constant factor, they both correspond to the greatest common divisor of $\mathfrak{u}_{1}$ and $\mathfrak{u}_{2}$. 


\subsection{The univariate polynomials $\mathfrak{u}_{1}, \mathfrak{u}_{2}$}

We explicitly perform the elimination step to pass from system $q=p_{1}=$ $p_{2}=0$ to

$$
\mathfrak{u}_{1}=\mathfrak{u}_{2}=0,
$$

where $\mathfrak{u}_{1}, \mathfrak{u}_{2}$ are the two univariate polynomials introduced in (27). For this purpose we produce an equivalent system $q=\tilde{p}_{1}=\tilde{p}_{2}=0$ where the $\tilde{p}_{j}$ are linear in one variable, say $\rho_{1}$.

Assume $q$ is not degenerate, i.e. $q_{2,0}, q_{0,2} \neq 0$. Indeed, here we use $q_{2,0} \neq 0$ only, while $q_{0,2} \neq 0$ is necessary for the similar construction relative to $\rho_{2}$. We write

$$
q\left(\rho_{1}, \rho_{2}\right)=\sum_{i, j=0}^{2} q_{i, j} \rho_{1}^{i} \rho_{2}^{j}=\sum_{h=0}^{2} b_{h}\left(\rho_{2}\right) \rho_{1}^{h},
$$

where

$$
b_{0}\left(\rho_{2}\right)=q_{0,2} \rho_{2}^{2}+q_{0,1} \rho_{2}+q_{0,0}, \quad b_{1}=q_{1,0}, \quad b_{2}=q_{2,0} .
$$

Moreover we write

$$
\begin{aligned}
& p_{1}\left(\rho_{1}, \rho_{2}\right)=\sum_{i, j=0}^{5} p_{i, j}^{(1)} \rho_{1}^{i} \rho_{2}^{j}=\sum_{h=0}^{4} a_{1, h}\left(\rho_{2}\right) \rho_{1}^{h}, \\
& p_{2}\left(\rho_{1}, \rho_{2}\right)=\sum_{i, j=0}^{5} p_{i, j}^{(2)} \rho_{1}^{i} \rho_{2}^{j}=\sum_{h=0}^{5} a_{2, h}\left(\rho_{2}\right) \rho_{1}^{h},
\end{aligned}
$$

for some polynomials $a_{k, h}$ whose degrees are described by the small circles used to construct Newton's polygons of $p_{1}, p_{2}$ in Figure 2. From $q=0$ we obtain

$$
\rho_{1}^{h}=\beta_{h} \rho_{1}+\gamma_{h}, \quad h=2,3,4,5
$$

where

$$
\beta_{2}=-\frac{b_{1}}{b_{2}}, \quad \gamma_{2}=-\frac{b_{0}}{b_{2}}
$$

and

$$
\beta_{h+1}=\beta_{h} \beta_{2}+\gamma_{h}, \quad \gamma_{h+1}=\beta_{h} \gamma_{2}, \quad h=2,3,4 .
$$

Inserting (30) into (28), 29) we obtain

$$
\tilde{p}_{j}\left(\rho_{1}, \rho_{2}\right)=\tilde{a}_{j, 1}\left(\rho_{2}\right) \rho_{1}+\tilde{a}_{j, 0}\left(\rho_{2}\right), \quad j=1,2
$$


where

$$
\begin{aligned}
& \tilde{a}_{1,1}=a_{1,1}+\sum_{h=2}^{4} a_{1, h} \beta_{h}, \quad \tilde{a}_{1,0}=a_{1,0}+\sum_{h=2}^{4} a_{1, h} \gamma_{h}, \\
& \tilde{a}_{2,1}=a_{2,1}+\sum_{h=2}^{5} a_{2, h} \beta_{h}, \quad \tilde{a}_{2,0}=a_{2,0}+\sum_{h=2}^{5} a_{2, h} \gamma_{h} .
\end{aligned}
$$
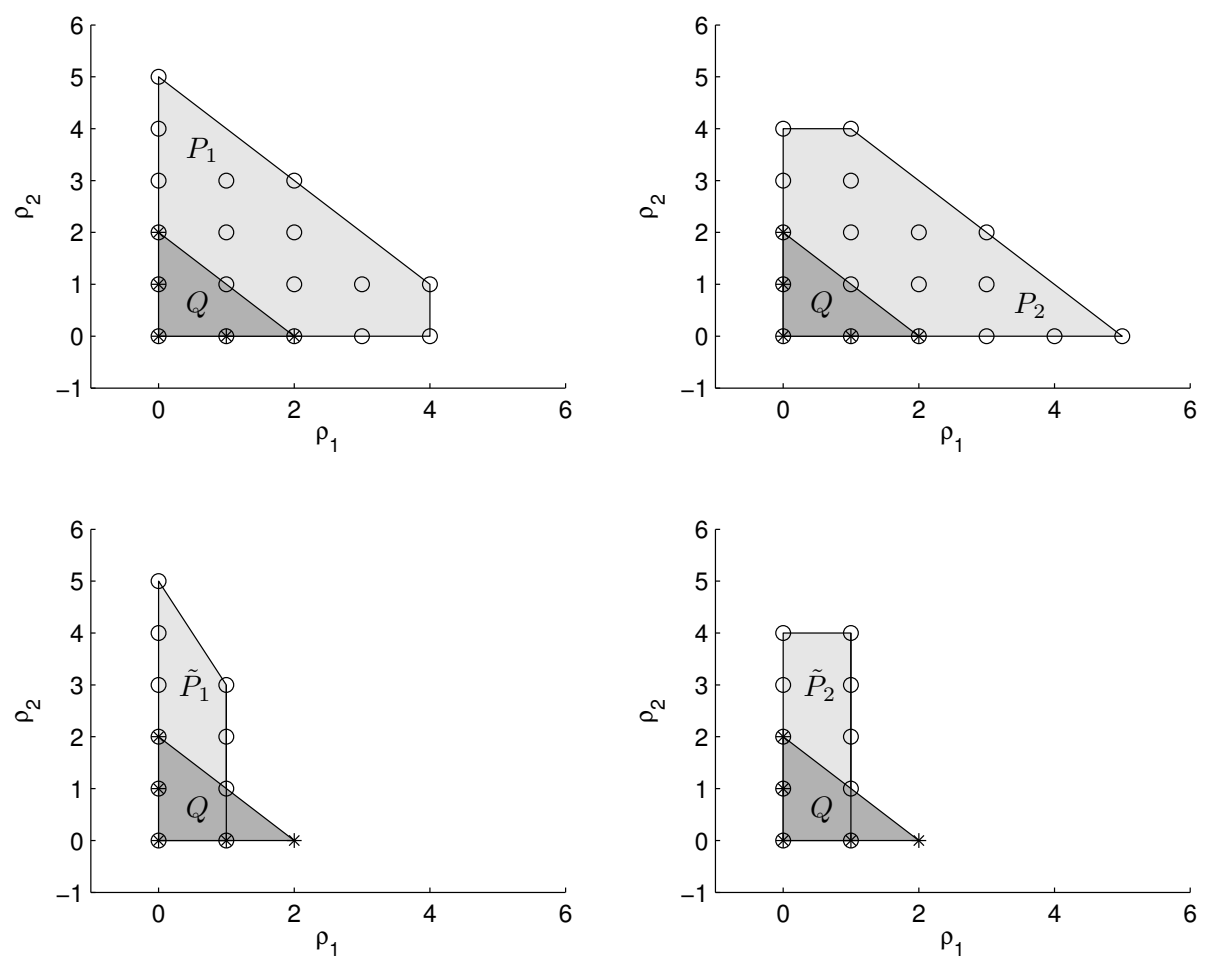

Figure 2: Newton's polygons $Q, P_{j}, \tilde{P}_{j}$ for the polynomials $q, p_{j}, \tilde{p}_{j}, j=$ 1,2 . The nodes with circles correspond to (multi-index) exponents of the monomials in $p_{j}, \tilde{p}_{j}$; the nodes with asterisks correspond to exponents of the monomials in $q$.

In Figure 2, bottom left and right, we draw Newton's polygons of $\tilde{p}_{1}, \tilde{p}_{2}$, which also describe the degrees of the polynomials $\tilde{a}_{k, h}$.

From (31) we get two expressions for $\rho_{1}$ :

$$
\rho_{1}=-\frac{\tilde{a}_{1,0}}{\tilde{a}_{1,1}}, \quad \rho_{1}=-\frac{\tilde{a}_{2,0}}{\tilde{a}_{2,1}} .
$$


By substituting these expressions into $q$ we obtain two univariate polynomials of degree 10 in the variable $\rho_{2}$ :

$$
\begin{aligned}
& \mathfrak{v}_{1}=q_{2,0} \tilde{a}_{1,0}^{2}-q_{1,0} \tilde{a}_{1,0} \tilde{a}_{1,1}+b_{0} \tilde{a}_{1,1}^{2}, \\
& \mathfrak{v}_{2}=q_{2,0} \tilde{a}_{2,0}^{2}-q_{1,0} \tilde{a}_{2,0} \tilde{a}_{2,1}+b_{0} \tilde{a}_{2,1}^{2} .
\end{aligned}
$$

Using the properties of resultants we find that

$$
\mathfrak{u}_{1}=q_{2,0}^{3} \mathfrak{v}_{1}, \quad \mathfrak{u}_{2}=q_{2,0}^{4} \mathfrak{v}_{2}
$$

\subsection{Non-degeneracy conditions}

We list below the conditions on the data $\mathcal{A}_{1}, \mathcal{A}_{2}, \mathbf{q}_{1}, \mathbf{q}_{2}, \dot{\mathbf{q}}_{1}, \dot{\mathbf{q}}_{2}$ that we used in the previous sections. These conditions generically hold.

1. $\mathbf{E}_{1} \cdot \mathbf{D}_{1} \times \mathbf{D}_{2}, \mathbf{E}_{2} \cdot \mathbf{D}_{1} \times \mathbf{D}_{2} \neq 0$, so that $q$ is a quadratic polynomial both in $\rho_{1}$ and $\rho_{2}$. An interpretation of these relations is given in [3]. Moreover, these conditions imply:

i) $\mathbf{D}_{1} \times \mathbf{D}_{2} \neq \mathbf{0}$, so that we can compute $\dot{\rho}_{j}=\dot{\rho}_{j}\left(\rho_{1}, \rho_{2}\right)$ from system (10). This condition also implies $\mathbf{D}_{1}, \mathbf{D}_{2} \neq \mathbf{0}$, which in turn yield $\mathbf{r}_{1}, \mathbf{r}_{2} \neq \mathbf{0}$ for all $\rho_{1}, \rho_{2}$;

ii) $\mathbf{e}_{1}^{\rho} \times \mathbf{e}_{1}^{\perp} \cdot \mathbf{q}_{1}, \mathbf{e}_{2}^{\rho} \times \mathbf{e}_{2}^{\perp} \cdot \mathbf{q}_{2} \neq 0$, which are used to define $\rho_{1}^{\prime \prime}, \rho_{2}^{\prime \prime}$ respectively;

iii) $\mathbf{e}_{1}^{\rho} \times \mathbf{e}_{2}^{\rho} \cdot \mathbf{q}_{1}, \mathbf{e}_{1}^{\rho} \times \mathbf{e}_{2}^{\rho} \cdot \mathbf{q}_{2} \neq 0$, which are used to define $\rho_{2}^{\prime}, \rho_{1}^{\prime}$ respectively.

2. $\mathbf{q}_{1} \times \mathbf{q}_{2} \neq \mathbf{0}, \rho_{j}^{\prime} \neq \rho_{j}^{\prime \prime}, \rho_{j}^{\prime \prime} \neq 0, j=1,2$. These conditions imply $\mathbf{r}_{1} \times \mathbf{r}_{2} \neq \mathbf{0}$, and also $\mathbf{r}_{1}, \mathbf{r}_{2}, \boldsymbol{\Delta}_{r} \neq \mathbf{0}$, for all $\rho_{1}, \rho_{2}$.

3. $\mathbf{e}_{j}^{\rho} \times \mathbf{e}_{j}^{\perp} \cdot \dot{\mathbf{q}}_{j} \neq 0$, so that $\dot{\mathbf{r}}_{j} \neq \mathbf{0}$ for all $\rho_{j}, \dot{\rho}_{j}$, with $j=1,2$.

4. $\boldsymbol{\Delta}_{q} \cdot \mathbf{e}_{1}^{\rho} \times \mathbf{e}_{2}^{\rho} \neq 0$, so that $\left\{\mathbf{e}_{1}^{\rho}, \mathbf{e}_{2}^{\rho}, \boldsymbol{\Delta}_{r}\right\}$ forms a basis of $\mathbb{R}^{3}$. This condition implies $\mathbf{e}_{1}^{\rho} \times \mathbf{e}_{2}^{\rho} \neq \mathbf{0}$, so that the maximal total degree for the components of equation (15) is 6 .

5. $p_{1}\left(P_{2}\right), p_{2}\left(P_{1}\right) \neq 0$, so that $P_{1}, P_{2}$ are not solutions of (17). 


\section{$6 \quad$ Selecting the solutions}

After computing all the solutions of 17 we can select the ones with both entries $\rho_{1}, \rho_{2}$ real and positive and compute the corresponding values of $\dot{\rho}_{1}, \dot{\rho}_{2}$. However, since equations (9) impose only some of the laws of the two-body dynamics, we expect that some of the remaining solutions yield vectors $\left(\rho_{1}, \rho_{2}, \dot{\rho}_{1}, \dot{\rho}_{2}, u_{1}, u_{2}\right)$ which do not solve (7).

In this section we characterize the spurious solutions, and propose some algorithms to select the good ones. To decide which solutions can be accepted we suggest to use one of the two methods introduced in [3], [4]. They take into account the errors in the observations, which can be represented by $4 \times 4$ covariance matrices $\Gamma_{1}, \Gamma_{2}$ of the attributables. We recall that the first method relies on the computation of a norm referring to some compatibility conditions, see 3], Section 5. The second method requires to compute a covariance matrix for the candidate preliminary orbits, which is used for the attribution algorithm, see [4], Sections 7, 8. The new formulas for the covariance matrix are provided in Section 6.2 .

\subsection{Spurious solutions}

We consider real solutions of (9) that do not solve the intermediate system (8), and solutions of (8) that do not solve (7). In the first case the spurious solutions do not satisfy

$$
\boldsymbol{\Delta}_{K} \cdot \boldsymbol{\Delta}_{r}+\left(\frac{1}{2}\left|\dot{\mathbf{r}}_{1}\right|^{2}-\frac{\mu}{\left|\mathbf{r}_{1}\right|}\right)\left|\boldsymbol{\Delta}_{r}\right|^{2}=0
$$

Concerning the second case we prove the following:

Proposition 1. Each real solution of (8) which does not solve (7) fulfills

$$
\left|\mathbf{L}_{1}-\mathbf{L}_{2}\right|=2
$$

Proof. Using $\mathbf{c}_{1}=\mathbf{c}_{2}$, the second equation in (8) can be written as

$$
\left(\mathbf{L}_{1}-\mathbf{L}_{2}\right)=\mu \frac{\left(\left|\mathbf{L}_{1}\right|^{2}-\left|\mathbf{L}_{2}\right|^{2}\right)}{2|\mathbf{c}|^{2}} \mathbf{r}_{2}
$$



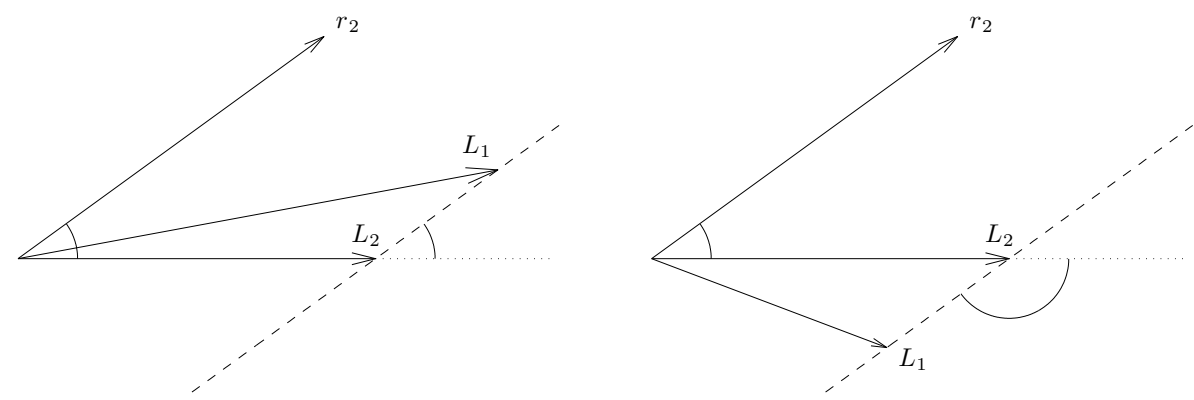

Figure 3: Left: case $\left|\mathbf{L}_{1}\right|>\left|\mathbf{L}_{2}\right|$. Right: case $\left|\mathbf{L}_{1}\right|<\left|\mathbf{L}_{2}\right|$.

where $\mathbf{c}$ is the common value of the angular momentum. The conservation of Laplace-Lenz vector and energy is in general not guaranteed. However, we note that if $\mathbf{c}_{1}=\mathbf{c}_{2}$ and $\left|\mathbf{L}_{1}\right|=\left|\mathbf{L}_{2}\right|$, then (8), (32) imply $\mathbf{L}_{1}=\mathbf{L}_{2}$ and $\mathcal{E}_{1}=\mathcal{E}_{2}$.

If $\left|\mathbf{L}_{1}\right|>\left|\mathbf{L}_{2}\right|$, the vectors $\mathbf{L}_{1}-\mathbf{L}_{2}, \mathbf{r}_{2}$ have the same orientation. Passing to the norms in (32) and substituting

$$
\left|\mathbf{r}_{2}\right|=\frac{|\mathbf{c}|^{2}}{\mu\left(1+\left|\mathbf{L}_{2}\right| \cos \theta_{2}\right)},
$$

where $\theta_{2}$ is the angle between $\mathbf{L}_{2}$ and $\mathbf{r}_{2}$, we obtain

$$
\left|\mathbf{L}_{1}-\mathbf{L}_{2}\right|=\frac{\left|\mathbf{L}_{1}\right|^{2}-\left|\mathbf{L}_{2}\right|^{2}}{2\left(1+\left|\mathbf{L}_{2}\right| \cos \theta_{2}\right)}
$$

Using relation

$$
\left|\mathbf{L}_{1}\right|^{2}-\left|\mathbf{L}_{2}\right|^{2}=\left|\mathbf{L}_{1}-\mathbf{L}_{2}\right|^{2}+2\left|\mathbf{L}_{1}-\mathbf{L}_{2}\right|\left|\mathbf{L}_{2}\right| \cos \theta_{2}
$$

and rearranging the terms we get $\left|\mathbf{L}_{1}-\mathbf{L}_{2}\right|=2$.

If $\left|\mathbf{L}_{1}\right|<\left|\mathbf{L}_{2}\right|$ the vectors $\mathbf{L}_{1}-\mathbf{L}_{2}, \mathbf{r}_{2}$ have opposite orientation, and we obtain $\left|\mathbf{L}_{1}-\mathbf{L}_{2}\right|=-2$, which is impossible. In fact in this case we have (see Figure 30

$$
\left|\mathbf{L}_{1}\right|^{2}-\left|\mathbf{L}_{2}\right|^{2}=\left|\mathbf{L}_{1}-\mathbf{L}_{2}\right|^{2}+2\left|\mathbf{L}_{1}-\mathbf{L}_{2}\right|\left|\mathbf{L}_{2}\right| \cos \left(\pi-\theta_{2}\right) .
$$

Since system (8) is generically inconsistent, the spurious solutions discussed in Proposition [1] usually do not occur. 


\subsection{Covariance of the solutions}

We introduce the vectors

$$
\mathbf{A}=\left(\mathcal{A}_{1}, \mathcal{A}_{2}\right), \quad \mathbf{R}=\left(\rho_{1}, \dot{\rho}_{1}, \rho_{2}, \dot{\rho}_{2}\right)
$$

and the map

$$
\boldsymbol{\Phi}(\mathbf{R}, \mathbf{A})=\left(\begin{array}{c}
\mathbf{c}_{1}-\mathbf{c}_{2} \\
\boldsymbol{\Delta}_{K} \times \Delta_{r} \cdot \mathbf{e}_{1}^{\rho}
\end{array}\right) .
$$

Assume we have a pair of attributables $\overline{\mathbf{A}}=\left(\overline{\mathcal{A}}_{1}, \overline{\mathcal{A}}_{2}\right)$, with covariance matrices $\Gamma_{1}, \Gamma_{2}$, at epochs $\bar{t}_{1}, \bar{t}_{2}$. For each vector $\overline{\mathbf{R}}$ such that

$$
\boldsymbol{\Phi}(\overline{\mathbf{R}}, \overline{\mathbf{A}})=\mathbf{0}, \quad \operatorname{det} \frac{\partial \Phi}{\partial \mathbf{R}}(\overline{\mathbf{R}}, \overline{\mathbf{A}}) \neq 0
$$

there exists a map $\mathbf{A} \mapsto \mathbf{R}(\mathbf{A})$ with $\mathbf{\Phi}(\mathbf{R}(\mathbf{A}), \mathbf{A})=\mathbf{0}$, defined in a neighborhood of $\overline{\mathbf{A}}$, with $\mathbf{R}(\overline{\mathbf{A}})=\overline{\mathbf{R}}$. Following [4], we consider the map $\boldsymbol{\Psi}$ defined by $\boldsymbol{\Phi}=\boldsymbol{\Psi} \circ \mathcal{T}_{\text {att }}^{c a r}$, where $\mathcal{T}_{\text {att }}^{\text {car }}$ is the transformation from attributable coordinates $\mathbf{E}_{a t t}$ to Cartesian coordinates $\mathbf{E}_{\text {car }}$ at the two epochs. Then we can use the same scheme as in [4] (Section 7) to compute the covariance matrix of the Cartesian coordinates at epoch $\bar{t}_{1}$ through the formula

$$
\Gamma_{c a r}^{(1)}=\frac{\partial E_{c a r}^{(1)}}{\partial \mathbf{A}} \Gamma_{\mathbf{A}}\left[\frac{\partial E_{c a r}^{(1)}}{\partial \mathbf{A}}\right]^{T}, \quad \Gamma_{\mathbf{A}}=\left[\begin{array}{cc}
\Gamma_{1} & 0 \\
0 & \Gamma_{2}
\end{array}\right]
$$

with the derivatives $\frac{\partial E_{\text {car }}^{(1)}}{\partial \mathbf{A}}$ evaluated at $\mathbf{A}=\overline{\mathbf{A}}$.

The only differences with respect to [4] are in the term $\frac{\partial \Psi}{\partial \mathbf{E}_{c a r}}$, which in this case is given by

$$
\frac{\partial \Psi}{\partial \mathbf{E}_{c a r}}=\left[\begin{array}{cccc}
-\widehat{\hat{\mathbf{r}}_{1}} & \widehat{\mathbf{r}_{1}} & \widehat{\hat{\mathbf{r}}_{2}} & -\widehat{\mathbf{r}_{2}} \\
\frac{\partial p_{1}}{\partial \mathbf{r}_{1}} & \frac{\partial p_{1}}{\partial \dot{\mathbf{r}}_{1}} & \frac{\partial p_{1}}{\partial \mathbf{r}_{2}} & \frac{\partial p_{1}}{\partial \dot{\mathbf{r}}_{2}}
\end{array}\right]
$$

where we use the hat map

$$
\mathbb{R}^{3} \ni\left(u_{1}, u_{2}, u_{3}\right)=\mathbf{u} \mapsto \widehat{\mathbf{u}}=\left[\begin{array}{ccc}
0 & -u_{3} & u_{2} \\
u_{3} & 0 & -u_{1} \\
-u_{2} & u_{1} & 0
\end{array}\right]
$$


To compute the derivatives of $p_{1}=\boldsymbol{\Delta}_{K} \times \boldsymbol{\Delta}_{r} \cdot \mathbf{e}_{1}^{\rho}$ we take advantage of the following relation, which holds at the solutions of $p_{1}=0$ :

$$
\frac{\partial p_{1}}{\partial \mathbf{E}_{c a r}}=\frac{1}{\rho_{1}} \frac{\partial p_{1}^{*}}{\partial \mathbf{E}_{c a r}}
$$

Here $p_{1}^{*}=\boldsymbol{\Delta}_{K} \times \boldsymbol{\Delta}_{r} \cdot \mathbf{v}_{1}$, with $\mathbf{v}_{1}=\mathbf{r}_{1}-\mathbf{q}_{1}$, and

$$
\begin{aligned}
\frac{\partial p_{1}^{*}}{\partial \mathbf{r}_{1}}= & \frac{1}{2}\left(\left|\dot{\mathbf{r}}_{2}\right|^{2}-\left|\dot{\mathbf{r}}_{1}\right|^{2}\right) \mathbf{q}_{1} \times \mathbf{r}_{2}-\left(\dot{\mathbf{r}}_{1} \times \boldsymbol{\Delta}_{r} \cdot \mathbf{v}_{1}\right) \dot{\mathbf{r}}_{1} \\
& -\left(\dot{\mathbf{r}}_{1} \cdot \mathbf{r}_{1}\right)\left[-\mathbf{q}_{1} \times \dot{\mathbf{r}}_{1}+\mathbf{r}_{2} \times \dot{\mathbf{r}}_{1}\right]+\left(\dot{\mathbf{r}}_{2} \cdot \mathbf{r}_{2}\right)\left[-\mathbf{q}_{1} \times \dot{\mathbf{r}}_{2}+\mathbf{r}_{2} \times \dot{\mathbf{r}}_{2}\right], \\
\frac{\partial p_{1}^{*}}{\partial \dot{\mathbf{r}}_{1}=} & -\left(\mathbf{r}_{1} \times \mathbf{r}_{2} \cdot \mathbf{v}_{1}\right) \dot{\mathbf{r}}_{1}-\left(\dot{\mathbf{r}}_{1} \times \boldsymbol{\Delta}_{r} \cdot \mathbf{v}_{1}\right) \mathbf{r}_{1}-\left(\dot{\mathbf{r}}_{1} \cdot \mathbf{r}_{1}\right) \boldsymbol{\Delta}_{r} \times \mathbf{v}_{1}, \\
\frac{\partial p_{1}^{*}}{\partial \mathbf{r}_{2}=} & -\frac{1}{2}\left(\left|\dot{\mathbf{r}}_{2}\right|^{2}-\left|\dot{\mathbf{r}}_{1}\right|^{2}\right) \mathbf{q}_{1} \times \mathbf{r}_{1}-\left(\dot{\mathbf{r}}_{1} \cdot \mathbf{r}_{1}\right) \dot{\mathbf{r}}_{1} \times \mathbf{v}_{1} \\
& +\left(\dot{\mathbf{r}}_{2} \times \boldsymbol{\Delta}_{r} \cdot \mathbf{v}_{1}\right) \dot{\mathbf{r}}_{2}+\left(\dot{\mathbf{r}}_{2} \cdot \mathbf{r}_{2}\right) \dot{\mathbf{r}}_{2} \times \mathbf{v}_{1}, \\
\frac{\partial p_{1}^{*}=}{\partial \dot{\mathbf{r}}_{2}=} & \left(\mathbf{r}_{1} \times \mathbf{r}_{2} \cdot \mathbf{v}_{1}\right) \dot{\mathbf{r}}_{2}+\left(\dot{\mathbf{r}}_{2} \times \boldsymbol{\Delta}_{r} \cdot \mathbf{v}_{1}\right) \mathbf{r}_{2}+\left(\dot{\mathbf{r}}_{2} \cdot \mathbf{r}_{2}\right) \boldsymbol{\Delta}_{r} \times \mathbf{v}_{1} .
\end{aligned}
$$

\section{Including the $J_{2}$ effect}

If we want to compute preliminary orbits of space debris, we have to take into account the effect of the oblateness of the Earth ( $J_{2}$ effect), which gives rise to a perturbation of the two-body dynamics. Following [2] and [4] we can use an iterative scheme to include the $J_{2}$ effect in the determination of preliminary orbits with the two-body integrals. We consider the equations

$$
R_{c} \mathbf{c}_{1}=\mathbf{c}_{2}, \quad R_{L} \mathbf{L}_{1}=\mathbf{L}_{2}, \quad \mathcal{E}_{1}=\mathcal{E}_{2}, \quad u_{1}^{2}\left|\mathbf{r}_{1}\right|^{2}=\mu^{2}, \quad u_{2}^{2}\left|\mathbf{r}_{2}\right|^{2}=\mu^{2},
$$

where the rotation matrices

$$
R_{c}=R_{\Delta \Omega}^{\hat{z}}, \quad R_{L}=R_{\omega_{1}+\Delta \omega}^{\mathbf{c}_{2}} R_{\Delta \Omega}^{\hat{z}} R_{-\omega_{1}}^{\mathbf{c}_{1}}
$$

are defined through the angles

$$
\Delta \omega=\omega_{2}-\omega_{1}, \quad \Delta \Omega=\Omega_{2}-\Omega_{1}
$$

In (33) we use $R_{\theta}^{\mathbf{v}}$ to denote the rotation by the angle $\theta$ around the axis defined by the vector $\mathbf{v}$. 
Following the same steps of Section 5 we consider the intermediate equation

$$
\mu R_{L} \mathbf{L}_{1}-\mathcal{E}_{1} \mathbf{r}_{2}=\mu \mathbf{L}_{2}-\mathcal{E}_{2} \mathbf{r}_{2}
$$

which can be written

$$
\boldsymbol{\Delta}_{K}^{L}+\left(\frac{1}{2}\left|\dot{\mathbf{r}}_{1}\right|^{2}-u_{1}\right) \boldsymbol{\Delta}_{r}^{L}=\mathbf{0}
$$

where

$$
\boldsymbol{\Delta}_{K}^{L}=R_{L} \mathbf{K}_{1}-\mathbf{K}_{2}, \quad \boldsymbol{\Delta}_{r}^{L}=R_{L} \mathbf{r}_{1}-\mathbf{r}_{2} .
$$

Then we can eliminate the dependence on $u_{1}$ by vector product with $\boldsymbol{\Delta}_{r}^{L}$. We end up with the system

$$
R_{c} \mathbf{c}_{1}=\mathbf{c}_{2}, \quad \boldsymbol{\Delta}_{K}^{L} \times \boldsymbol{\Delta}_{r}^{L}=\mathbf{0} .
$$

Note that system (34) is not polynomial due to the presence of the rotation matrices $R_{c}, R_{L}$, that depend on the orbital elements. We can search for solutions of (34) by considering the solutions of (9), i.e. assuming $R_{L}=$ $R_{c}=I$, as first guesses of the iterative method. Inserting these solutions into $R_{L}$ and $R_{c}$, system (34) becomes polynomial, and we can solve it like in the unperturbed case. We iterate the procedure and consider the solutions at convergence, if any. Some care must be taken in selecting the solutions at each iteration.

Similarly to 16 we have

$\boldsymbol{\Delta}_{K}^{L} \times \boldsymbol{\Delta}_{r}^{L}=\frac{1}{2}\left(\left|\dot{\mathbf{r}}_{2}\right|^{2}-\left|\dot{\mathbf{r}}_{1}\right|^{2}\right) R_{L} \mathbf{r}_{1} \times \mathbf{r}_{2}-\left(\dot{\mathbf{r}}_{1} \cdot \mathbf{r}_{1}\right) R_{L} \dot{\mathbf{r}}_{1} \times \Delta_{r}^{L}+\left(\dot{\mathbf{r}}_{2} \cdot \mathbf{r}_{2}\right) \dot{\mathbf{r}}_{2} \times \Delta_{r}^{L}$.

By developing the expressions of $R_{L} \mathbf{r}_{1} \times \mathbf{r}_{2}, R_{L} \dot{\mathbf{r}}_{1} \times \boldsymbol{\Delta}_{r}^{L}, \dot{\mathbf{r}}_{2} \times \boldsymbol{\Delta}_{r}^{L}$ as polynomials in $\rho_{1}, \rho_{2}$ we find that the monomials in $\Delta_{K}^{L} \times \Delta_{r}^{L}$ with the highest degree (i.e. 6) are all multiplied by $R_{L} \mathbf{e}_{1}^{\rho} \times \mathbf{e}_{2}^{\rho}$. Then we can project the second equation in (34) onto the vectors $R_{L} \mathbf{e}_{1}^{\rho}, \mathbf{e}_{2}^{\rho}$, to obtain two polynomial equations $p_{j}\left(\rho_{1}, \rho_{2}\right)=0, j=1,2$. As before, $p_{1}$ and $p_{2}$ have degree 5 . The range rates can be eliminated from equation $R_{c} \mathbf{c}_{1}=\mathbf{c}_{2}$, to get a quadratic polynomial $q$ analogous to (12).

An important difference with respect to Section 5 is that in this case the system $p_{1}=p_{2}=q=0$ is generically inconsistent, as can be checked by a numerical test. Nevertheless we can choose either $p_{1}=q=0$ or $p_{2}=q=0$ as polynomial equations for the linkage. 

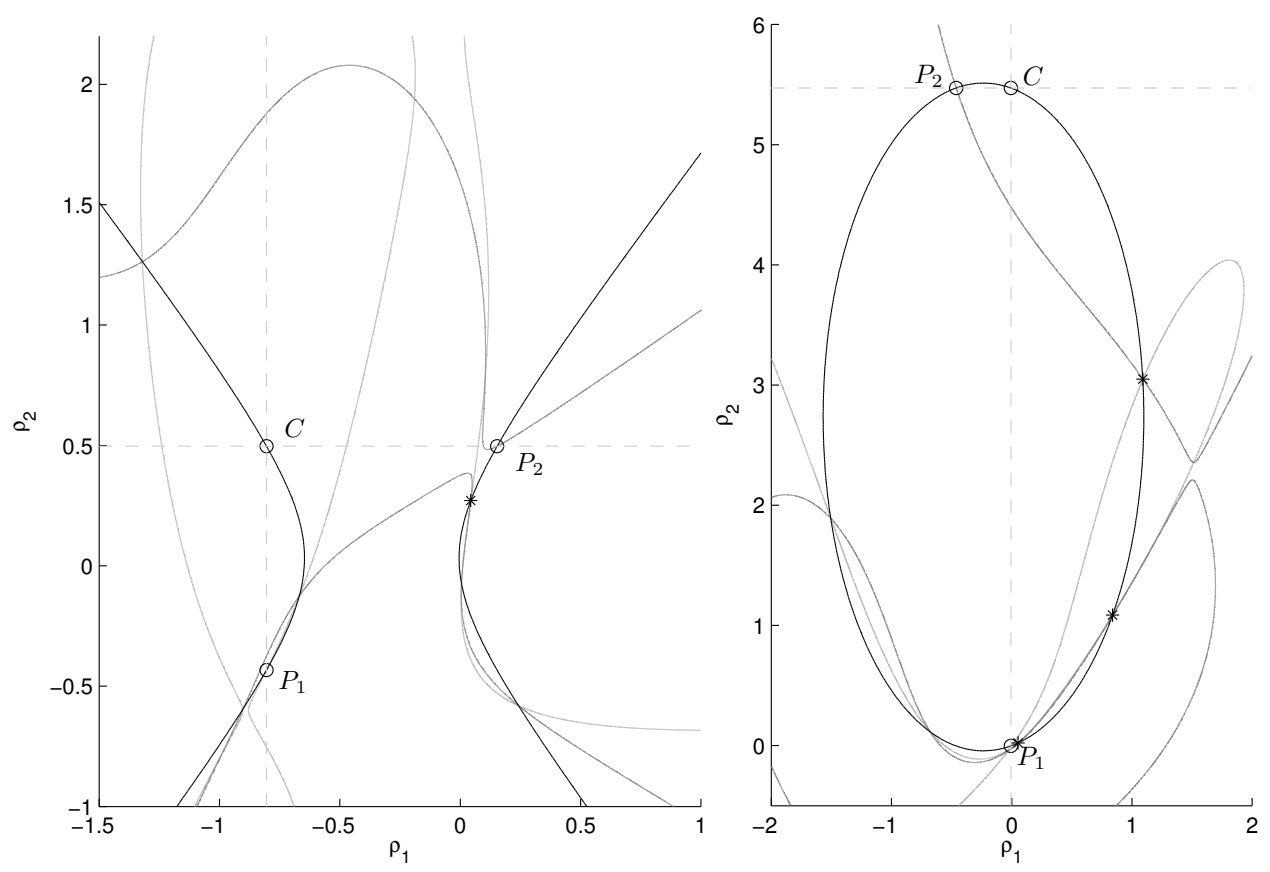

Figure 4: Left: Intersection of curves defined by $q=p_{1}=p_{2}=0$ for (101955) Bennu. Right: the same for (100000) Astronautica.

\section{Numerical tests}

We describe the results of two numerical tests, one for a near-Earth asteroid, the other for a main belt. The first object is (101955) Bennu. We link 5 observations made on September 11, 1999 together with 11 observations made on March 30, 2000. After discarding non-real and non-positive solutions, we are left with only one pair

$$
\left(\rho_{1}, \rho_{2}\right)=(0.04379,0.27132) .
$$

The second object is the main belt asteroid (100000) Astronautica. We link 10 observations made on October 17 and 19, 2003 together with 4 observations made on May 9, 2005. In this case we are left with the pairs

$$
\left(\rho_{1}, \rho_{2}\right)=(0.05240,0.02179), \quad(0.83897,1.08648), \quad(1.09052,3.04874) .
$$

The third pair is discarded because it yields an unbounded orbit at epoch $\bar{t}_{2}$. The values of the penalty (see [4]) for the first and second solution, computed 


\begin{tabular}{l|c|c|c|c|c|c|c} 
asteroid & MJD & $a$ & $e$ & $I$ & $\Omega$ & $\omega$ & $\ell$ \\
\hline$(101955)$ & 51432.4 & 1.1316 & 0.2058 & 5.9895 & 2.1229 & 65.2425 & 303.8601 \\
& 51633.4 & 1.1306 & 0.2039 & 5.9895 & 2.1229 & 66.4329 & 107.6598 \\
\hline$(100000)_{1}$ & 52930.2 & 1.0365 & 0.0277 & 1.0298 & 202.3124 & 275.0221 & 268.4023 \\
& 53499.3 & 1.0367 & 0.0305 & 1.0298 & 202.3124 & 308.8393 & 73.7641 \\
\hline$(100000)_{2}$ & 52930.2 & 1.8725 & 0.0768 & 20.8665 & 186.7970 & 197.8094 & 344.8383 \\
& 53499.3 & 1.8754 & 0.0862 & 20.8665 & 186.7970 & 197.7172 & 198.9997
\end{tabular}

Table 1: Keplerian elements found for (101955) Bennu, and for (100000) Astronautica.

by attribution, are $\chi_{4}=1224012.479$ and $\chi_{4}=1.497$ respectively, therefore we select the second solution.

In Figure 4 we draw the curves $q=0$ (black), $p_{1}=0$ (light gray), $p_{2}=0$ (dark gray). The dashed straight lines correspond to $c_{11}=0$ (vertical), $c_{22}=0$ (horizontal). The computed pairs $\left(\rho_{1}, \rho_{2}\right)$ are marked with asterisks.

In Table 1 we show for both asteroids the Keplerian elements at the two mean epochs $\bar{t}_{1}, \bar{t}_{2}$ that we find with this method. For asteroid (100000), we indicate with the labels 1,2 the two bounded orbits that we can compute.

\section{Acknowledgments}

The work is partially supported by the Marie Curie Initial Training Network Stardust, FP7-PEOPLE-2012-ITN, Grant Agreement 317185.

\section{A Appendix}

We suggest a way to discard pairs of attributables which are not likely to fulfill the requirements to be linked. This filter is based on the angular momentum equation $\mathbf{c}_{1}=\mathbf{c}_{2}$.

\section{A.1 Filtering pairs of attributables}

The equation $q=0$ represents a conic $Q$ in the plane $\rho_{1} \rho_{2}$. We can decide to accept only pairs $\left(\rho_{1}, \rho_{2}\right)$ inside a square $\mathcal{R}=\left[\rho_{\min }, \rho_{\max }\right] \times\left[\rho_{\min }, \rho_{\max }\right]$, with $0<\rho_{\min }<\rho_{\max }$. We check whether the conic $Q$ does intersect $\mathcal{R}$. 
First consider the case $Q$ is unbounded (hyperbola or parabola). In this case it is sufficient to check whether the conic intersects the boundary $\partial \mathcal{R}$.

If $Q$ is bounded (ellipse or circle) this check is not enough: it can happen that $Q \cap \mathcal{R} \neq \emptyset$, with $Q$ lying totally inside $\mathcal{R}$.

Here we sketch an algorithm for this filter. Consider the four straight lines

$$
r_{j}=\left\{\left(\rho_{1}, \rho_{2}\right): \rho_{j}=\rho_{\min }\right\}, \quad r_{j+2}=\left\{\left(\rho_{1}, \rho_{2}\right): \rho_{j}=\rho_{\max }\right\}, \quad \text { for } j=1,2 .
$$

First compute the intersections of the conic with each line $r_{j}, j=1 \ldots 4$, if any. If an intersection lies on the segment of some $r_{j}$ belonging to $\partial \mathcal{R}$ we accept the pair of attributables and continue the linkage procedure. If not, we check whether $Q$ is bounded or unbounded.

If $Q$ is unbounded we discard the pairs of attributables. If $Q$ is bounded, we can check whether $Q$ is totally inside $\mathcal{R}$ by computing the coordinates of the center of the conic. If the center is inside $\mathcal{R}$ we accept the pair provided $Q$ has no intersections with any $r_{j}$, otherwise we reject it.

\section{References}

[1] Cox, D., Little, J., O'Shea, D.: Ideals, Varieties, and Algorithms, Springer (2005).

[2] Farnocchia, D., Tommei, G., Milani, A., Rossi, A.: Innovative methods of correlation and orbit determination for space debris, CMDA 107/1-2, 169-185 (2010).

[3] Gronchi, G. F., Dimare, L., Milani, A.: Orbit determination with the two-body integrals, CMDA 107/3, 299-318 (2010).

[4] Gronchi, G. F., Farnocchia, D., Dimare, L.: Orbit determination with the two-body integrals. II, CMDA 110/3, 257-270 (2011).

[5] Milani, A., Sansaturio, M. E., Chesley, S. R.: The Asteroid Identification Problem IV: Attributions, Icarus 151, 150-159 (2001).

[6] Milani, A., Gronchi, G. F.: The theory of Orbit Determination, Cambridge Univ. Press (2010).

[7] Taff, L. G., Hall, D. L.: The use of angles and angular rates. I - Initial orbit determination, CMDA 16, 481-488 (1977). 